\title{
Using a hybrid genetic algorithm-simulated annealing algorithm for fuzzy programming of reservoir operation
}

\author{
Yu-Chen Chiu, ${ }^{1}$ Li-Chiu Chang ${ }^{2}$ and Fi-John Chang ${ }^{1 *}$ \\ ${ }^{1}$ Department of Bioenvironmental Systems Engineering, National Taiwan University, Taipei, Taiwan, ROC \\ ${ }^{2}$ Department of Water Resources and Environmental Engineering, Tamkang University
}

\begin{abstract}
:
We present a novel approach for optimizing reservoir operation through fuzzy programming and a hybrid evolution algorithm, i.e. genetic algorithm (GA) with simulated annealing (SA). In the analysis, objectives and constraints of reservoir operation are transformed by fuzzy programming for searching the optimal degree of satisfaction. In the hybrid search procedure, the GA provides a global search and the SA algorithm provides local search. This approach was investigated to search the optimizing operation scheme of Shihmen Reservoir in Taiwan. Monthly inflow data for three years reflecting different hydrological conditions and a consecutive 10-year period were used. Comparisons were made with the existing M-5 reservoir operation rules. The results demonstrate that: (1) fuzzy programming could effectively formulate the reservoir operation scheme into degree of satisfaction $\alpha$ among the users and constraints; (2) the hybrid GA-SA performed much better than the current M-5 operating rules. Analysis also found the hybrid GA-SA conducts parallel analyses that increase the probability of finding an optimal solution while reducing computation time for reservoir operation. Copyright $\ 2007$ John Wiley \& Sons, Ltd.
\end{abstract}

KEY WORDS genetic algorithm; simulated annealing; hybrid GA-SA; reservoir operation; fuzzy programming

Received 5 September 2005; Accepted 28 April 2006

\section{INTRODUCTION}

Water shortages in Taiwan are a regular occurrence owing to a monsoonal rainfall of wet and dry periods. Because approximately $75 \%$ of the rainfall is concentrated in typhoons occurring from May to October and this rainfall runs off in a matter of days, water supplies depend on reservoir retention. An increasing population and coupled economic growth requires advanced management techniques to assure water is available when needed. There are 66 reservoirs in Taiwan, with most classified as small or middle sized, having a storage capacity of less than $5 \times 10^{6} \mathrm{~m}^{3}$. These reservoirs are experiencing a serious reduction in storage capacity caused by sedimentation related to landslides during typhoons. Unless reservoir operation can be improved, water shortages are expected to occur more frequently. Reservoir operation has emerged as a critical issue in the sustainable utilization of water resources in Taiwan.

Reservoir operations in Taiwan must consider rainfall seasonality and typhoon influences, multiple water uses, and legal and other constraints. Because the decisionmaking process involves uncertainty and inaccuracies, water resource managers mainly rely on operation rules, which were obtained through simulation and optimization techniques. Applications of optimization in reservoir operation are not new. Dynamic programming (DP),

* Correspondence to: Fi-John Chang, Department of Bioenvironmental Systems Engineering and Hydrotech Research Institute, National Taiwan University, Taipei, Taiwan, ROC. E-mail: changfj@ntu.edu.tw originally developed by Bellman (1957) and applied by Hall and Buras (1961), has been used to solve the problems of reservoir operation. Many methods have been proposed to improve and/or implement DP for reservoir operation (e.g. Datta and Houck, 1984; Trezos and Yeh, 1987; Archibald et al., 1996; Chang et al., 2002; Chaves et al., 2003). The major disadvantage of DP is that computation times grow exponentially with the dimension of the state space (number of decision variables). Dimensionality issues limit the use of DP in exploring an optimal long-term operating schedule for effective water distribution.

In recent years, new methods of optimization have emerged. Search methods based on natural evolution mechanisms, such as genetic algorithms (GAs) and simulated annealing (SA) have been shown to have advantages over classical optimization methods and have become widely used for solving a number of hydrological and water resource problems (Oliveira and Loucks, 1997; Pardo-Igúzquiza, 1998; Wardlaw and Sharif, 1999; Chang and Chang, 2001; Chang et al., 2005). GAs are based on the process of genetic change in living organisms, whereas SA is based on thermodynamic principles. The main advantages of these algorithms are their flexibility, ease of implementation, broad applicability and the potential of finding a nearoptimal solution for many problems. Both GAs and SA support general-purpose optimization methods. GA approaches provide a global search through a systematic and parallel manner. SA search methods, which 
can theoretically converge to the global optimum solution with unit probability (Kirkpatrick et al., 1983), provide a local solution. Nevertheless, both methods usually required longer computation times (Wang and Zheng, 1998).

In this study, we propose the use of fuzzy programming to formulate the reservoir operation for multiple water uses in different hydrological conditions and a hybrid GA-SA algorithm for optimizing the operation schemes. The idea of constructing a hybrid GA-SA algorithm is mainly inspired by combining these two algorithms in order to facilitate the exhaustive and parallel treatment of optimization problems, to increase the probability of finding an optimal solution, and to reduce the computation time required to reach a solution.

\section{METHODOLOGIES}

Reservoir operation in Taiwan must consider hydrological conditions, especially typhoon influences, multiple water uses, and other constraints. To deal with uncertainty and inaccuracies in the decision-making process of reservoir operation, fuzzy programming is proposed to formulate the problem into a degree of satisfaction $\alpha$ among the users and constraints. The hybrid GA-SA algorithm is then used to search the optimal reservoir operation. The GA can perform a global search (population) and the SA algorithm can perform a local search (single point). To demonstrate this idea, the GA, SA and hybrid GA-SA algorithms were used to search 12 monthly water releases of Shihmen Reservoir in Taiwan. Monthly inflow data for three years reflecting different hydrological conditions were used to investigate these algorithms' performances (efficiency and effectiveness). For further evaluation of the hybrid GA-SA method in searching for long-term reservoir operation performance, we implemented the method over a consecutive 10-year period.

Because the M-5 rule curves are the official way for guiding reservoir operation, it is crucial to learn about its performances in various hydrological conditions as a base for comparison. Consequently, a reference operational scheme was identified from the existing M-5 operational rules. In the following sections, fuzzy set theory, which is used to transform the objective and constraints of reservoir operation into fuzzy programming, is first briefly introduced and the optimizing algorithms (GA, $\mathrm{SA}$, and hybrid GA-SA) are given.

\section{Fuzzy set theory}

Because reservoir operation involves targets and values that are often subjective and change with time, evaluating all objectives and constraints is a complex undertaking, if not impossible. To deal with this uncertainty, fuzzy set theory was used to identify targets and values that were then subject to natural system search techniques. Fuzzy set theory, developed by Zadeh (1965), provides a mathematical basis for representing linguistic vagueness. In the last decade, the area of fuzzy set theory has seen a surge in research, particularly in hydrology, where research has focused on rainfall-runoff relationships (Maskey et al., 2004), rainfall forecasting (Yu et al., 2000), and reservoir operation (Chang et al., 2002; Dubrovin et al., 2002, Mousavi et al., 2004).

Bellman and Zadeh (1970) developed a fuzzy programming approach to address optimization problems with fuzzy objectives and constraints to develop a decisionmaking model. In this approach, the fuzzy objective and constraints are transformed through membership functions into crisp objectives and constraints (Zimmermann, 1976; Wang, 1997). The optimum solution in fuzzy programming will be the intersection of the degrees of membership of the objective $\mu_{\bar{G}}(x)$ and constraints $\mu_{\bar{C}}(x)$ and is defined as follows:

$$
\mu_{\bar{D}}(x)=\mu_{\bar{G}}(x) \wedge \mu_{\bar{C}}(x)=\min \left(\mu_{\bar{G}}(x), \mu_{\bar{C}}(x)\right)
$$

Because many possible solutions are possible, fuzzy set theory, specifically fuzzy programming, was used to determine the optimal solution with the maximum membership value:

$$
\mu_{\bar{Z}}(x)=\max \mu_{\bar{D}}(x)
$$

The fuzzy programming can then be expressed as

$$
\begin{aligned}
& C^{\mathrm{T}} X \geq Z \\
& A_{i} X \leq b_{i} \\
& X_{i} \geq 0
\end{aligned}
$$

where $X$ is the decision vector, $C^{\mathrm{T}}$ and $A_{i}$ are objective and constraints matrices respectively, $b_{i}$ is the maximum desired levels, and $Z$ is a given aspiration level. The degrees of membership of the objective $\mu_{0}(x)$ and constraints $\mu_{i}(x)$ can be defined by the following membership functions:

$$
\begin{gathered}
\mu_{0}(x)=\left\{\begin{array}{cc}
C^{\mathrm{T}} X>Z \\
1-\frac{Z-C^{\mathrm{T}} X}{p_{0}} & Z-p_{0} \leq C^{\mathrm{T}} X \leq Z \\
0 & C^{\mathrm{T}} X<Z-p_{0}
\end{array}\right. \\
\mu_{i}(x)=\left\{\begin{array}{cc}
1 & A_{i} X<b_{i} \\
1-\frac{A_{i} X-b_{i}}{p_{i}} & b_{i} \leq A_{i} X \leq b_{i}+p_{i} \quad i=1,2, \ldots, m \\
0 & A_{i} X>b_{i}+p_{i}
\end{array}\right.
\end{gathered}
$$

where $p_{0}$ and $p_{i}$ are tolerances of the objective and the $i$ th constraint.

Let $\alpha$ represent the satisfaction of the objective and constraints; the above problem can then be integrated as a maximizing satisfaction and transformed into the following mathematical programming:

$$
\begin{aligned}
& \max \alpha \\
& \text { s.t. } \mu_{0}=1-\frac{Z-C^{\mathrm{T}} X}{p_{0}} \geq \alpha \\
& \mu_{i}=1-\frac{A_{i} X-b_{i}}{p_{i}} \geq \alpha, \quad i=1,2, \ldots, m
\end{aligned}
$$




$$
\begin{aligned}
& \alpha, \mu_{i} \in[0,1] \\
& X \geq 0
\end{aligned}
$$

Therefore, the task of fuzzy programming is to determine $\alpha$, the degree of satisfaction for the problem.

The optimum solution in fuzzy programming will then be searched by the GA, SA, and hybrid GA-SA optimizing algorithms.

\section{Genetic algorithm methods}

The GA is a search and optimization technique based on the mechanics of natural selection and genetics (Holland, 1992). According to Goldberg (1989), the major differences between GAs and traditional optimization methods are: (i) GAs use an evolving coding parameter set, not the parameters themselves; (ii) GAs search a population of points, not a single point; (iii) GAs use probabilistic transition rules, not deterministic rules. The GA is an adaptive and robust method for searching the optimum solution to a complex problem, although it may not necessarily lead to the best possible solution.

Reproduction. Reproduction is the procedure by which chromosomes are chosen in the next generation. A popular approach is fitness proportionate selection (Goldberg 1989), in which the probability $P_{i}$ of an individual $i$ being selected is given by

$$
P_{i}=\frac{f_{i}}{\sum_{i=1}^{n} f_{i}}
$$

where $f_{i}$ is the fitness of $i$ and $n$ is the population size.

Crossover. As in living organisms, the GA attempts to preserve useful mutations so that, as new strings are created, the best are preserved. The crossover operator exchanges building blocks between two strings that perform well. The number of strings in which material is exchanged is controlled by the crossover probability, usually in the range of 0.5-1.0. In this study, the blend crossover (BLX- $\alpha$ ) (Eshelman and Schaffer, 1993; Chang and Chen, 1998) is used.

Mutation. In living organisms, mutation changes the characteristics of genetic material in a chromosome to sustain genetic diversity in the population. Mutation is made occasionally with small probability. A random position of a random string is selected and is replaced by another character from the alphabet; for example, in the binary coding, this simply means changing ones to zeros and vice versa. A mutation operator of the real-coded GA is that specific element of a chromosome randomly that randomly jumps in the search space if it has an exactly equal chance of undergoing the mutative process.

\section{Simulated annealing}

$\mathrm{SA}$ is analogous to the annealing process used in metallurgy, where a metal object is heated to near its melting temperature and then cooled slowly. This allows metal atoms to align themselves, crystallize and attain a minimum energy state. The SA algorithm, first proposed by Kirkpatrick et al. (1983), is a multipurpose heuristic searching technique for optimizing functions of many variables, and will evaluate a sequence of local optima in search of the global optimum. The SA algorithm superimposes a neighbourhood structure on the finite but large space of feasible solutions. Given a current solution $X_{\text {cur }}$, a candidate solution $X_{\text {can }}$ is drawn randomly from the corresponding neighbourhood. This new solution $X_{\text {can }}$ will be accepted to substitute the original solution $X_{\text {cur }}$ subject to either improvement of the objective or a random experiment with an acceptance probability given by the Metropolis rule $\exp (-\Delta C / \beta)$, where $\Delta C=$ $C\left(X_{\text {can }}\right)-C\left(X_{\text {cur }}\right)$ is the difference of the cost function values and $\beta$ is the control parameter corresponding to temperature. According to the Metropolis rule, the SA would accept a worse solution in a given fraction of cases, and the fraction of worse solutions gradually approaches zero as the temperature decreases. If the new solution is accepted, then it will become the current point; otherwise, a new starting point from the current one is attempted. The number of searching points at a temperature can be set and seen as the length of the Markov chain.

The solution points in SA satisfy the Boltzmann distribution, so that at any given temperature the probability of obtaining a lower cost ending point is higher when the iteration is sufficiently large. However, in any implementation of the algorithm the Markov chain is of finite length. Therefore, asymptotic convergence can only be approximate.

The main advantage of SA is its general ease of solution. Solving a specific problem by SA, the user needs to determine the annealing schedule $T^{K}$, control parameter $\beta$, length of Markov chain $L_{\max }$, and a neighbourhood structure.

\section{Hybrid genetic algorithm-simulated annealing algorithm}

The GA-SA process consists of coupling a GA with SA in an iterative manner. In the initial GA phase, genetic operators are used to identify an interim solution, which is then used in SA analysis. The GA generates a set of initial random solutions and then uses selection, crossover and mutation operators for few generations $N_{\text {initial }}$.

After $N_{\text {initial }}$ generations, all of the latest solutions are sent to the SA for further improvement. Because SA is a single-insertion neighbourhood scheme, the $\mathrm{SA}$ is executed for each solution from the GA. Once the SA is performed for all solutions in the latest generation of the GA, the best solutions obtained from SA are the solutions of the GA for the next generation. The GA and SA exchange continues until the required number of cycles is reached or the system has frozen. A flow chart of this two-stage searching technique is shown in Figure 1. Several important factors which could significantly influence the searching effectiveness and 


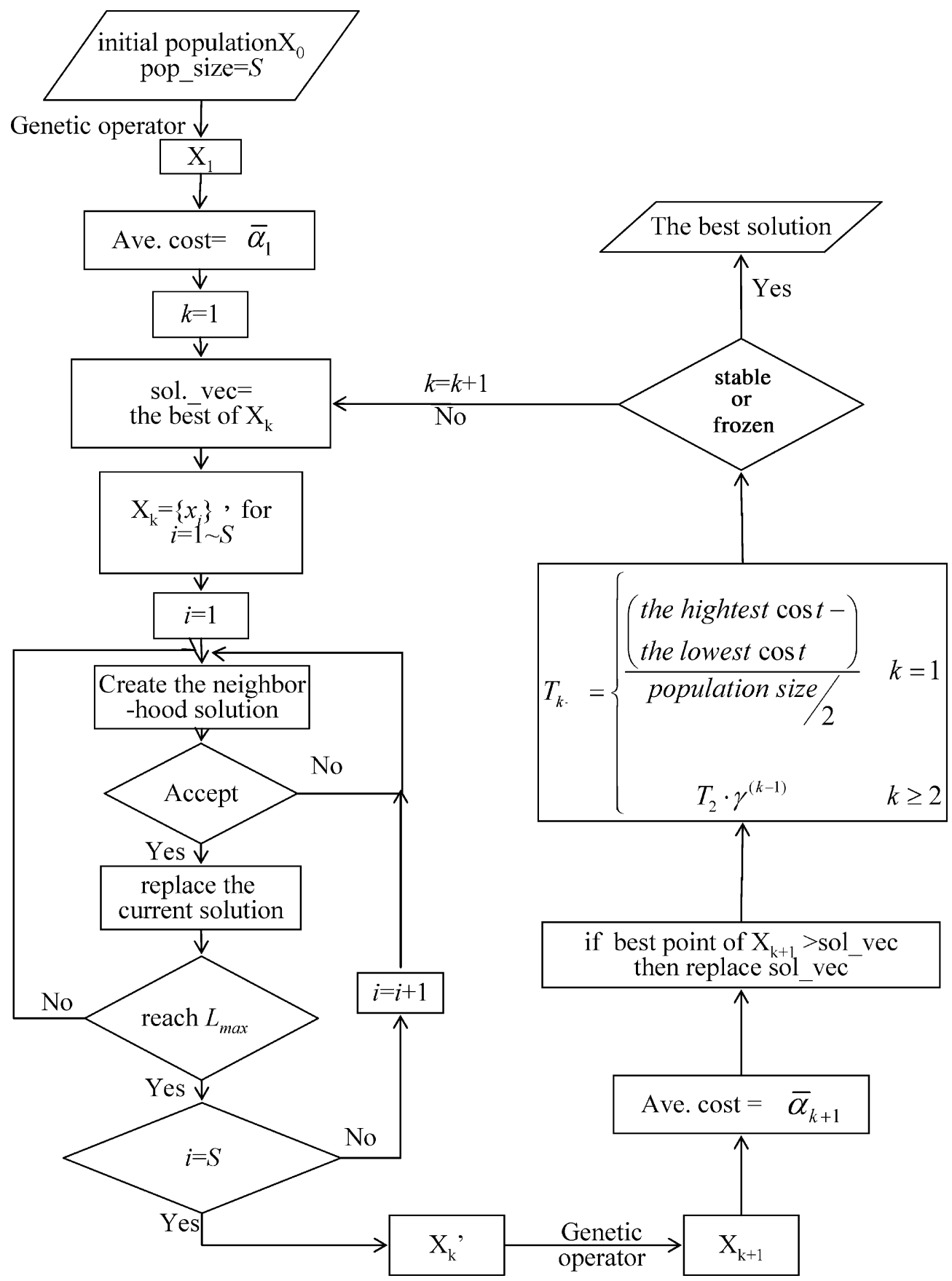

Figure 1. The flow chart of the hybrid GA-SA algorithm

efficiency through the searching process are given as follows.

Changed temperature. If the initial temperature $T_{0}$ is set to a high value, then the search route will result in high degree of randomness and will produce many redundant iterations in the course of SA (Dougherty and Marryott, 1991). Such a strategy is not used in our GA approach. Instead, the selection scheme of the GA is used. The hybrid algorithm starts with a randomly generated set of solutions (population $X_{0}$ ). Next, the GA is applied for few generations $N_{\text {initial }}$ to produce a new set of solutions (population $X_{1}$ ) by rejecting the higher cost offspring so that the average cost must be less than the random cost
$X_{0}$. Then, the $\mathrm{SA}$ is performed from $X_{1}$ with a given length $L_{\max }$ of iteration for each solution until the next generation $X_{1}^{\prime}$ is created. The initial temperature is set as a small value, $T_{0}=0.01$, and the subsequent temperatures are set as suggested by Lin et al. (1993):

$$
T_{k}=\left\{\begin{array}{cc}
T_{1} & k=1 \\
T_{1} \gamma^{(k-1)} & k \geq 2
\end{array}\right.
$$

where $T_{1}=$ (highest cost - lowest cost)/(populationsize/ $2), k$ is the temperature stage and $\gamma(=0.8)$ is the decrement ratio of temperature. 
Stopping rule (system frozen). When the average cost of each generation is about equal to that of the best generation, the system tends to convergence (or is frozen). In this study, we set the lowest temperature equal to 0.001. As $T_{k}$ is less than this temperature, the system is assumed to converge and the computing process can then be stopped. In fact, the objective function does not fluctuate widely in computing process; the search process was terminated after five times of temperature decrease.

\section{APPLICATION}

\section{Reservoir system}

The metropolitan area of Taipei, population 6.5 million, is located on the Tanshui River and its tributaries in northern Taiwan (Figure 2). A major tributary of the Tanshui River is the Tahan River, on which Shihmen Reservoir is located. Shihmen Reservoir is managed for multiple purposes, including municipal and industrial water supply, agriculture, flood control, and power production. The operations of Shihmen Reservoir are based on the M-5 rule curves, as shown in Figure 3. The upper, lower, and critical limit curves are used for irrigation, power generation, and flood control respectively. Based on the operating rule curves, the operating policy can be briefly described as follows.

1. When the water level is above the upper limit, the water release for hydropower generation should be increased to keep the water level near the upper limit.

2. When the water level is between the upper and lower limits, the release, including public use, irrigation water supply and hydropower generation, is under normal operating conditions.

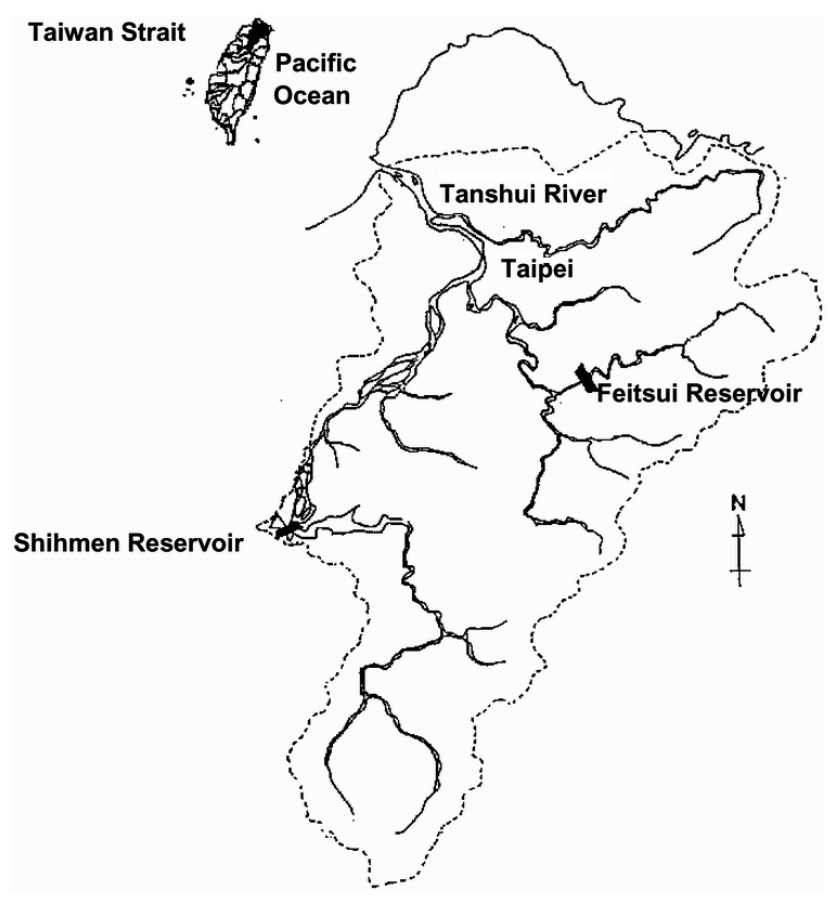

Figure 2. Location of Tanshui River basin, Taiwan

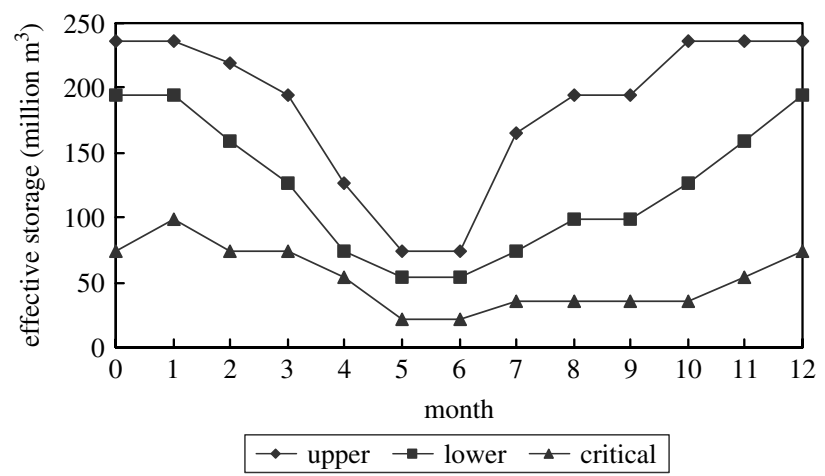

Figure 3. The M-5 rule curve of Shiman Reservoir

3. When the water level is between the lower and critical limits, public use and irrigation water can be supplied as usual, but the water release for hydropower generation must be cut back by $20 \%$.

4. When the water level is below critical limits, irrigation water must be cut back by $30 \%$ and the water release for hydropower generation must be reduced to the magnitude of public use plus irrigation water requirements only.

It appears that the operating rule defines the release within each year as a function of the existing storage level and overall release target amounts.

The monthly inflow of Shihmen Reservoir for three different hydrological years (1988, 1990, and 1993) was used in this analysis. Table I summarizes the monthly inflow and future water demand (i.e. 2010) where the demand was estimated based on the recorded monthly water use in 2001 multiplied by 1.1 .

\section{Modelling}

This analysis focused on storage and release, where storage is provided for flood control, power generation and recreation and releases are made to meet water supply demands. The dominant demands are for agriculture and

Table I. The monthly inflow and water demand

\begin{tabular}{lrrrr}
\hline & \multicolumn{2}{c}{ Monthly inflow $\left(10^{6} \mathrm{~m}^{3}\right)$} & $\begin{array}{r}\text { Monthly demand } \\
\left(10^{6} \mathrm{~m}^{3}\right)\end{array}$ \\
\cline { 2 - 4 } & \multicolumn{3}{c}{1988} & \\
\hline Jan & 37.24 & 59.47 & 34.12 & 65.58 \\
Feb & 36.43 & 46.67 & 24.61 & 92.00 \\
Mar & 52.38 & 78.76 & 70.94 & 119.37 \\
Apr & 133.77 & 273.45 & 96.24 & 107.21 \\
May & 82.94 & 100.32 & 84.22 & 109.11 \\
Jun & 87.73 & 219.50 & 159.29 & 108.82 \\
Jul & 40.12 & 111.68 & 69.76 & 132.68 \\
Aug & 62.67 & 637.19 & 70.90 & 131.63 \\
Sep & 114.88 & 571.39 & 34.24 & 112.34 \\
Oct & 258.45 & 76.47 & 40.97 & 111.39 \\
Nov & 92.73 & 48.57 & 27.06 & 78.12 \\
Dec & 36.35 & 33.00 & 26.14 & 50.37 \\
Total & 1035.69 & 2256.47 & 738.49 & 1218.62 \\
\hline
\end{tabular}


public water supply use. The objective function and constraints were formulated as:

Objective function.

$$
\min \left\{\frac{1}{5} \sum_{i=1}^{12}\left(\frac{S_{i}-X_{i}}{X_{i}}\right)^{2}+\frac{4}{5} \sum_{i=1}^{12}\left[\left(\frac{R_{i}-D_{i}}{D_{i}}\right)^{2} n_{i}\right]\right\}
$$

$Z$ is a given aspiration level and is set to be zero, $p_{0}$ is the tolerance of objective and is set as 12 , and $b_{i}$ and $p_{i}$ are the maximum elevation at which water should not be higher than this level and the tolerance of the $i$ th resource constraint respectively. Let $b_{i}$ be the upper curve of the M-5 rule curves and let $p_{i}$ be the difference between upper curve and full storage in this study. Therefore, the task of fuzzy programming is to determine $\alpha$ as

$$
\max \alpha=\max \left\{0, \min \left[\begin{array}{l}
1 \\
1-\frac{1}{p_{0}}\left\{\frac{1}{5} \sum_{i=1}^{12}\left(\frac{S_{i}-X_{i}}{X_{i}}\right)^{2}+\frac{4}{5} \sum_{i=1}^{12}\left[\left(\frac{R_{i}-D_{i}}{D_{i}}\right)^{2} n_{i}\right]\right\} \\
1-\frac{S_{i}-b_{i}}{p_{i}} \quad i=5,6,7,8,9
\end{array}\right]\right\}
$$

where $S_{i}$ is the effective storage of the $i$ th month, $R_{i}$ is the the release of the $i$ th month, $X_{i}$ is the the low rule in the M-5 curve of the $i$ th month, $D_{i}$ is the downstream demand of the $i$ th month, and $n_{i}$ is the number of cumulative shortage months.

\section{Constraints.}

$$
\begin{array}{ll}
\text { water balance } & R_{i}=S_{i-1}-S_{i}+q_{i} \\
\text { flood seasons } & 0 \leq S_{i} \leq X_{i}^{+} \\
\text {other seasons } & 0 \leq S_{i} \leq S_{\max } \quad \text { for } i=5,6,7,8,9 \\
& 0.9 S_{1} \leq S_{12} \leq 1.1 S_{1}
\end{array}
$$

where $q_{i}$ is the monthly inflow of the $i$ th month, $X_{i}^{+}$is the upper rule in the M-5 curve, and $S_{\max }$ is the effective storage of the reservoir.

As mentioned above, Shihmen Reservoir is a multipurpose reservoir; however, if one looks through the operation policy, it is easy to see that the main consideration is water supply for a different purpose. Consequently, the relative weights for the purpose of water storage versus water release are set as 1:4, as suggested by Chang and Chen (1991). Because reservoir operation involves targets and values that are often subjective and change with time, evaluating all objectives and constraints is a complex, if not impossible, undertaking. To deal with this uncertainty, fuzzy theory may provide the most appropriate methodology for modelling reservoir operation.

The fuzzy programming is rewritten as

$\max \alpha$

$$
\begin{array}{ll}
\text { s.t. } & \left\{\frac{1}{5} \sum_{i=1}^{12}\left(\frac{S_{i}-X_{i}}{X_{i}}\right)^{2}+\frac{4}{5} \sum_{i=1}^{12}\left[\left(\frac{R_{i}-D_{i}}{D_{i}}\right)^{2} n_{i}\right]\right\} \\
\leq Z+(1-\alpha) p_{0} &
\end{array}
$$

water balance $R_{i}=S_{i-1}-S_{i}+q_{i}$ for $i=1,2, \ldots, 12$

flood seasons $S_{i} \leq b_{i}+(1-\alpha) p_{i}$ for $i=5,6,7,8,9$

other seasons $0 \leq S_{i} \leq S_{\max }$ for $i=1,2,3,4,10,11,12$

$0.9 S_{1} \leq S_{12} \leq 1.1 S_{1}$

$0 \leq \alpha \leq 1$
For the purpose of comparison, the currently used M-5 operating rule curves for the operation of this reservoir are also evaluated.

\section{Results}

The performance of the proposed algorithms and the M-5 rule curve for the operation of Shihmen Reservoir were evaluated by using the degree of satisfaction $\alpha$ and the generalized shortage index GSI (Hsu, 1995), given by

$$
\mathrm{GSI}=\frac{100}{N} \sum_{i=1}^{N}\left(\frac{\mathrm{DPD}_{i}}{100 \times \mathrm{DY}_{i}}\right)^{2}
$$

where $N$ is number of sample years $(N=1), \mathrm{DY}_{i}$ is days of the $i$ th year $\left(\mathrm{DY}_{i}=365\right.$ or 366$)$ and $\mathrm{DPD}_{i}$ is the deficit percentage index of the $i$ th year, given by

$$
\mathrm{DPD}=\sum(\mathrm{DDR}(\%) \times \mathrm{NDC})
$$

where DDR is the daily deficit rate and NDC is number of days in a continuous deficit. In our case, because we use monthly data, the $\mathrm{DY}_{i}$ is changed to the number of months in a year, i.e. $\mathrm{DY}_{i}=12$, and DDR is changed to represent the monthly deficit rate and NDC is changed to represent the number of months in a continuous deficit. The GSI is an indicator of the social tolerance limits to water shortage that measures the yearly deficit rate. Under this criterion the model that produces the minimum GSI is the one with the better performance.

Table II gives the values of the parameters used in the GA-SA. These parameter values are also used in GA and $\mathrm{SA}$ analysis. Because the number of generations $N_{\mathrm{g}}$ in the GA phase could influence the convergence and quality of final solutions, $N_{\mathrm{g}}$ was determined in the following manner.

(1) The GA is performed for the first 30 generations with its maximum, minimum, and averaged values of the objective in each generation recorded (i.e. population size of 300). The results are shown in the Figure 4. It appears that the values of the objective are increasing rapidly in the first 10 generations and 
Table II. Parameters used in the GA-SA

\begin{tabular}{|c|c|c|c|c|}
\hline Algorithm & $\begin{array}{l}\text { First phase } \\
\text { GA }\end{array}$ & & $\begin{array}{c}\text { Second phase } \\
\text { SA }\end{array}$ & \\
\hline Parameters set & $\begin{array}{l}\text { Population size } S \\
\text { Crossover probability } P_{\mathrm{c}} \\
\text { Mutation probability } P_{\mathrm{m}} \\
\text { Number of generations } N_{\mathrm{g}}\end{array}$ & $\begin{aligned} & 300 \\
& 0 \cdot 8 \\
& 0 \cdot 05 \\
\leq & 10\end{aligned}$ & $\begin{array}{l}\text { Length of Markov chain } L_{\max } \\
\text { First stage } \\
\text { Initial temperature } T_{0} \\
\text { Other stage } \\
\text { Initial temperature } T_{1} \\
\text { Decrement ratio of temperature } \gamma\end{array}$ & $\begin{array}{l}\leq 100 \\
0.01{ }^{\circ} \mathrm{C} \\
\text { (highest cost }- \text { lowest cost }) /(300 / 2) \\
0.8\end{array}$ \\
\hline
\end{tabular}

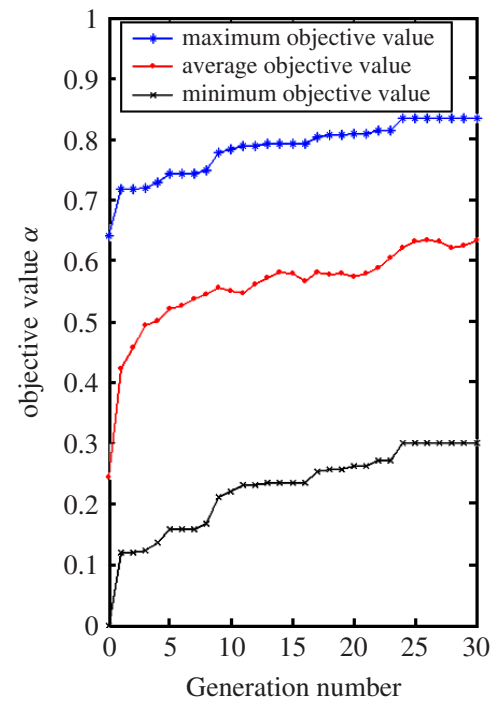

(a) 1988

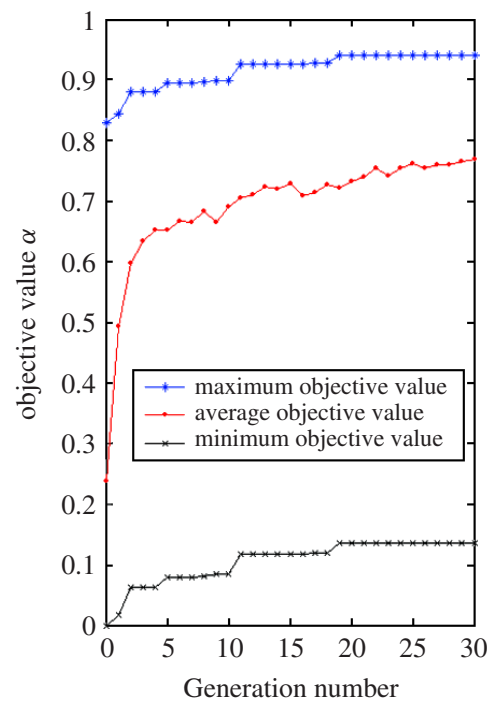

(b) 1990

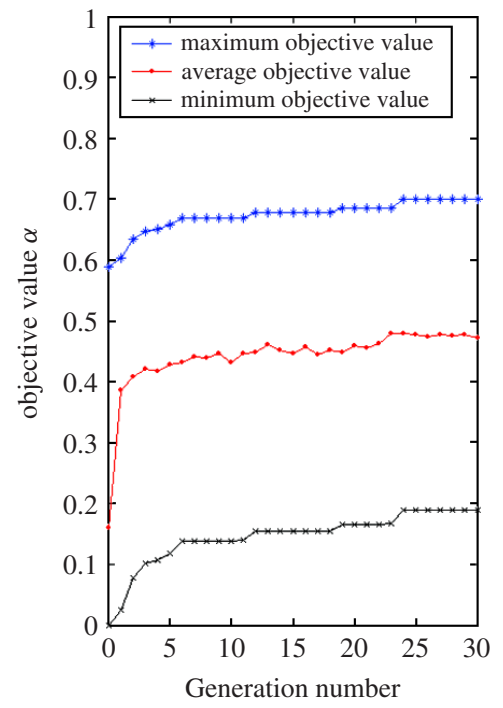

(c) 1993

Figure 4. The performances of the first 30 generations by the GA

increasing slightly after that. As a result, the number of generations $N_{\mathrm{g}}$ in the GA phase was set to less than 10 .

(2) Let $X_{k}^{\prime}$ be the set of solutions obtained by SA in the $k$ th stage and used to perform the GA operation. After $N_{\mathrm{g}}$ generations of the GA, we obtain a new set of solutions denoted as $X_{k+1}$.

Let $\overline{\alpha_{X_{k}^{\prime}}}$ and $\overline{\alpha_{X_{k+1}^{\prime}}}$ be the average cost of $X_{k}^{\prime}$ and $X_{k+1}$ respectively.

The degree of improvement $I$ in the average objective is defined as

$$
I=\frac{\overline{\alpha_{X_{k+1}}}-\overline{\alpha_{X_{k}^{\prime}}}}{\overline{\alpha_{X_{k}^{\prime}}}}
$$

As mentioned above, the values of the objective usually are rapidly increasing in the first 10 GA generations and slightly increasing after that. In order to make the hybrid GA-SA procedure more efficient and effective, the following strategy is proposed:

if $I \geq 1.5$ or $N_{\mathrm{g}}=10$, then stop the operator when

$k=1$ (first stage);

if $I \geq 0.05$ or $N_{\mathrm{g}}=10$, then stop the operator when

$$
k>1 \text {. }
$$

The results are summarized in Table III and Figures 5 and 6 for three different hydrological conditions. From these results we note that the GA, SA and GA-SA provide consistently superior performance for both degree of satisfaction $\alpha$ (above 60\%) and the GSI index when compared with the M-5 rule curve. The hybrid GA-SA method gives the highest satisfaction and lowest GSI values in all cases. On close examination of the results, we found that the traditional M-5 rule curves (the official way for guiding reservoir operation) did not provide suitable operating schemes in the three years investigated, where the GSI values in 1988 (average hydrological condition) and 1993 (drought year) are very high and the degree of satisfaction $\alpha=0$ in 1990 (wet year) and 1993. The results suggest that the M-5 rule curves should be adjusted to avoid a continuous water deficit in a drought year (even an average hydrological year) and a high risk of dam safety in a wet year. Table III also shows that there is high correlation between the degree of satisfaction and the GSI value: the higher the degree of satisfaction, the lower the GSI value. This result provides good evidence that the degree of satisfaction proposed in this study is a suitable criterion to evaluate reservoir operation with regard to water deficit and dam safety.

Figure 5 provides a comparison of methods, GA and SA, for the 1988, 1990 and 1993 hydrologic 
Table III. The search results of different methods

\begin{tabular}{llcrrr}
\hline Year & Item & M-5 rule & GA & SA & GA-SA \\
\hline 1988 & $\alpha$ & 0.46 & 0.85 & 0.83 & 0.86 \\
& GSI & 193.63 & 5.68 & 9.24 & 4.81 \\
1990 & $\alpha$ & 0 & 0.94 & 0.96 & 0.95 \\
& GSI & 2.45 & 0.47 & 0.78 & 0.20 \\
1993 & $\alpha$ & 0 & 0.63 & 0.66 & 0.73 \\
& GSI & 721.26 & 32.12 & 32.79 & 29.12 \\
\hline
\end{tabular}

conditions. The GA has a stepped response in the first 100 generations and then reaches a steady state. The SA shows variability at the outset then climbs to a steady state. Figure 6 presents the searching performance

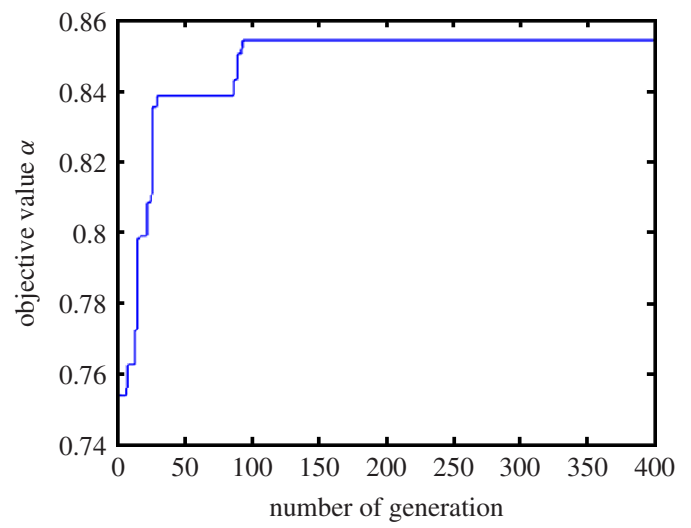

(a1) GA in 1988

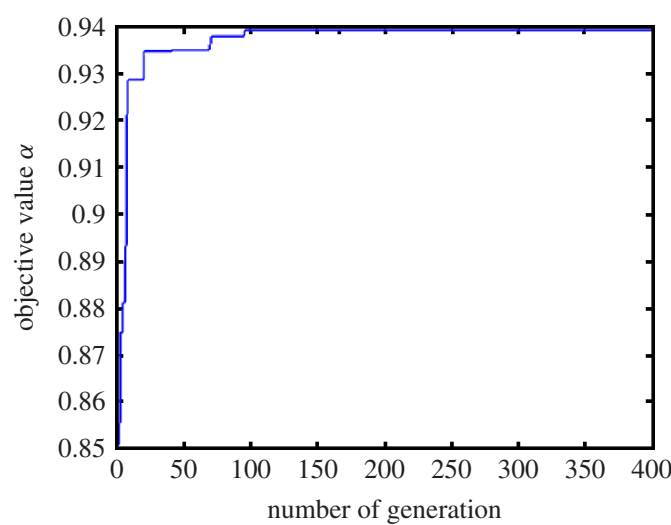

(b1) GA in 1990

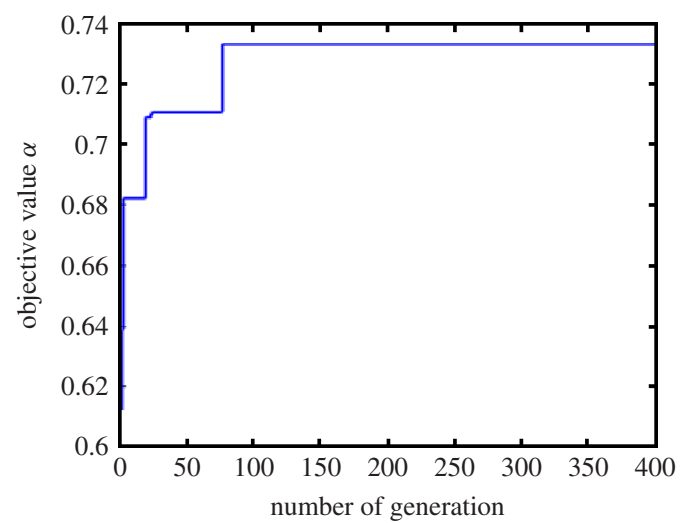

(c1) GA in 1993 through the GA-SA interaction stages. The ordinate is the degree of satisfaction. The upper abscissa indicates the number of temperature decreasing stages by SA and the lower abscissa represents the number of generations by the GA. After five stages of SA and less than 60 generations of the GA, the objective values reach a stable (converged) condition in all three cases. The hybrid GA-SA gives interesting and promising results, where the objective value is consistently increased in each GA-SA interacting stage. It appears that, in the first two or three GA-SA interacting stages, the GA contributes more than SA in terms of increasing the objective values, whereas the SA dominates the improvement of system performance in the later interacting stages in 1988 and 1990.

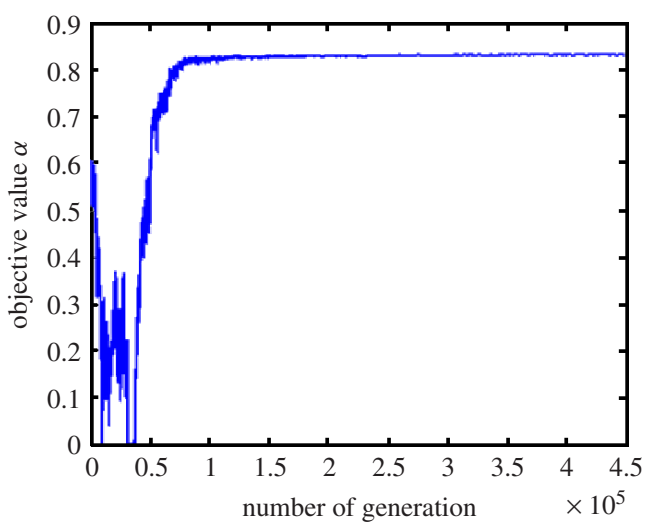

(a2) SA in 1988

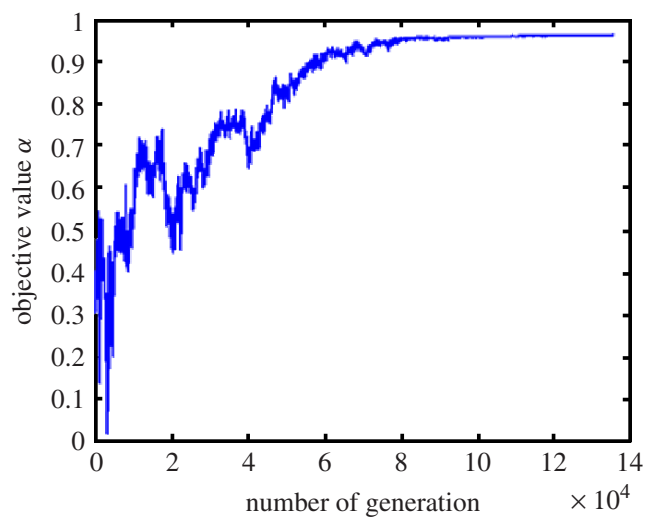

(b2) SA in 1990

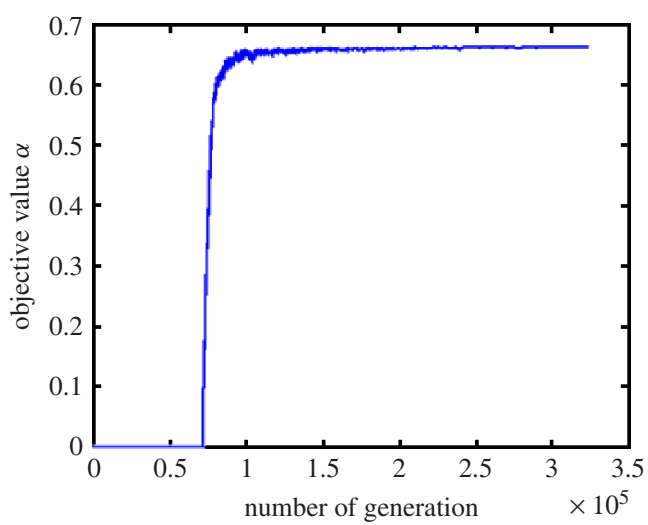

(c2) SA in 1993

Figure 5. The performances of the GA and SA through the searching processes in (a) 1988, (b) 1990 and (c) 1993 


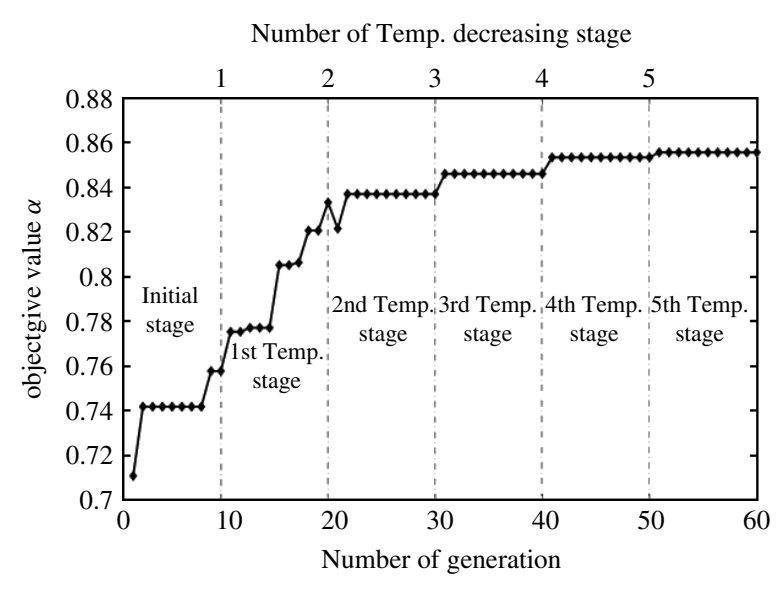

(a) GA-SA in 1988

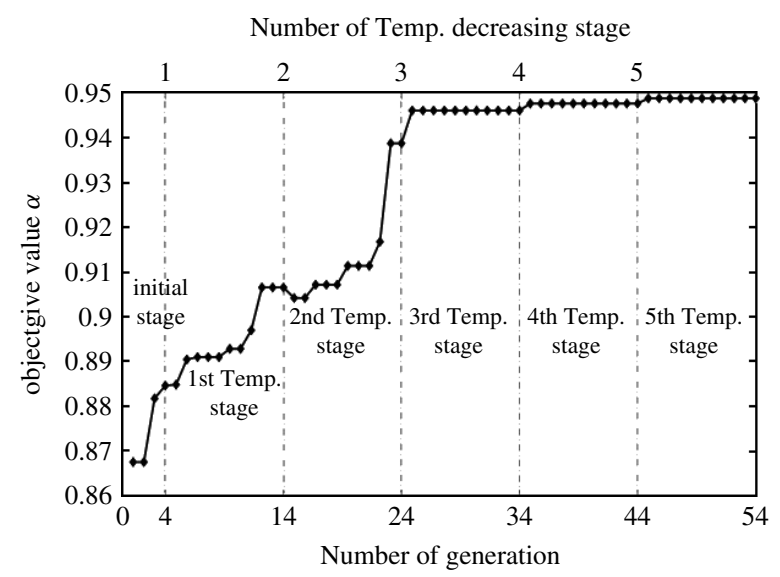

(b) GA-SA in 1990

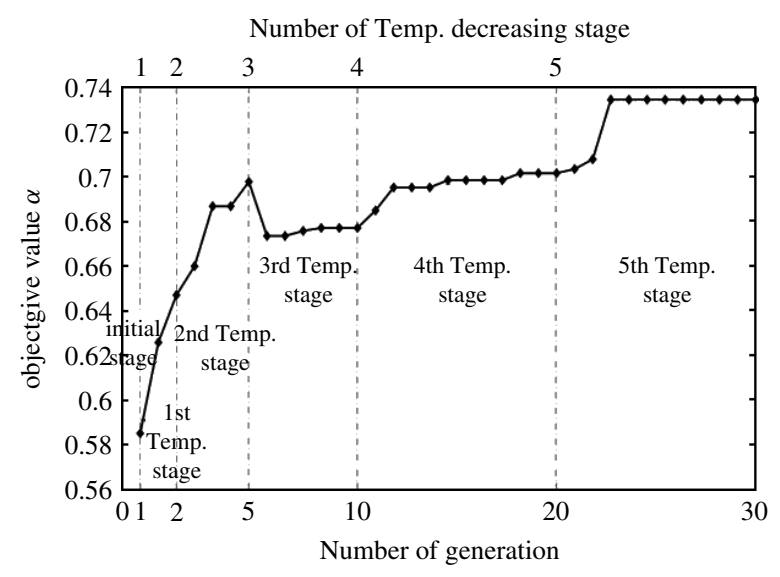

(c) GA-SA in 1993

Figure 6. The performance of the GA-SA through the searching processes in (a) 1988, (b) 1990 and (c) 1993

The number of searches (simulation times) is listed in Table IV. The searching times of the three methods are roughly estimated as follows.

1. GA: number of generations $\times$ population size, i.e. $400 \times 300$ in all three cases.

2. SA: number of iterations until a stable solution is reached which shows in the abscissa of part B Figure 5(a2), 5(b2), and 5(c2) for 1988, 1990, and 1993, respectively.
Table IV. The searching times of three methods in different years

\begin{tabular}{cccc}
\hline Year & \multicolumn{3}{c}{ Number of searches } \\
\cline { 2 - 4 } & GA & SA & GA-SA \\
\hline 1988 & $12 \times 10^{4}$ & $44.9 \times 10^{4}$ & $\leq 16.8 \times 10^{4}$ \\
1990 & $12 \times 10^{4}$ & $13.6 \times 10^{4}$ & $\leq 16.5 \times 10^{4}$ \\
1993 & $12 \times 10^{4}$ & $32.4 \times 10^{4}$ & $\leq 16.1 \times 10^{4}$ \\
\hline
\end{tabular}

3. GA-SA: number of generations $\times$ population size + number of temperature decreasing stages $\times$ number of iterations in each stage $\times$ population size (e.g. $60 \times 300+5 \times 100 \times 300=16.8 \times 10^{4}$ for 1988 ).

Table IV shows the searching times of the three methods in different years. The searching times of the hybrid GA-SA are slightly greater than the GA (the total computation time of the GA-SA is mainly contributed by SA), whereas SA usually takes more time to reach a stable solution. It is worth mentioning that the searching times of the hybrid GA-SA can be dramatically reduced if the population size is reduced. For instance, if one executes an SA search based on those of better solutions obtained from the GA process, e.g. upper 100 solutions in population size of 300 , one might obtain almost the same final objective values, whereas the number of searches could be reduced to $68000(60 \times 300+5 \times$ $100 \times 100=6.8 \times 10^{4}$ ) times for 1988 .

To testing and evaluate the hybrid GA-SA method further, we implemented a search on the long-term reservoir operation performance. Consecutive 10-year monthly historical inflow data were used. The search procedure, as above, is performed annually. Each year, 12 optimizing monthly releases and water storage levels were searched, and the last water storage level of the year was then propagated into the next year as its initial water storage level. For the purpose of comparison, the M-5 rule curves were also performed based on same inflow conditions and water requirements (as mentioned above). The results are presented in Table $\mathrm{V}$ and Figure 7. It appears that the hybrid GA-SA method outperforms the M-5 rule curves in terms of a much higher degree of satisfaction and lower GSI values in all 10 years. From Figure 7, we can easily see that the M-5 simulation method would produce a very high GSI value if the previous year was a drought year. This is mainly because the previous year, if it is a drought, will have a low water storage level at the end of that year and the low water level will propagate to the current year for the first four or five drought months. This would certainly exaggerate the drought phenomenon and greatly increase the GSI values.

\section{CONCLUSIONS}

In this study, Shihmen Reservoir operation was first transformed by fuzzy programming to identify the optimal 
Table V. The results of M-5 rules and GA-SA in 10 years

\begin{tabular}{|c|c|c|c|c|c|c|c|c|c|c|c|}
\hline & & 1988 & 1989 & 1990 & 1991 & 1992 & 1993 & 1994 & 1995 & 1996 & 1997 \\
\hline Inflow $\left(10^{6} \mathrm{~m}^{3}\right)$ & & 1036 & 1488 & 2256 & 1138 & 1910 & 738 & 1885 & 885 & 1456 & 1656 \\
\hline \multirow[t]{2}{*}{ GSI } & M-5 rules & 194 & 432 & 257 & $81 \cdot 0$ & 143 & $9 \cdot 84$ & 487 & $7 \cdot 78$ & 255 & 186 \\
\hline & GA-SA & 4.81 & $1 \cdot 80$ & $0 \cdot 60$ & $8 \cdot 19$ & 0.05 & $35 \cdot 8$ & $6 \cdot 00$ & 22.7 & 4.55 & $6 \cdot 87$ \\
\hline \multirow[t]{2}{*}{$\alpha$} & M-5 rules & 0.76 & 0 & 0 & $0 \cdot 8$ & 0 & 0.54 & 0 & 0.69 & 0 & 0 \\
\hline & GA-SA & $0 \cdot 86$ & 0.86 & 0.94 & 0.78 & 0.95 & $0 \cdot 64$ & $0 \cdot 82$ & 0.79 & 0.69 & 0.85 \\
\hline
\end{tabular}

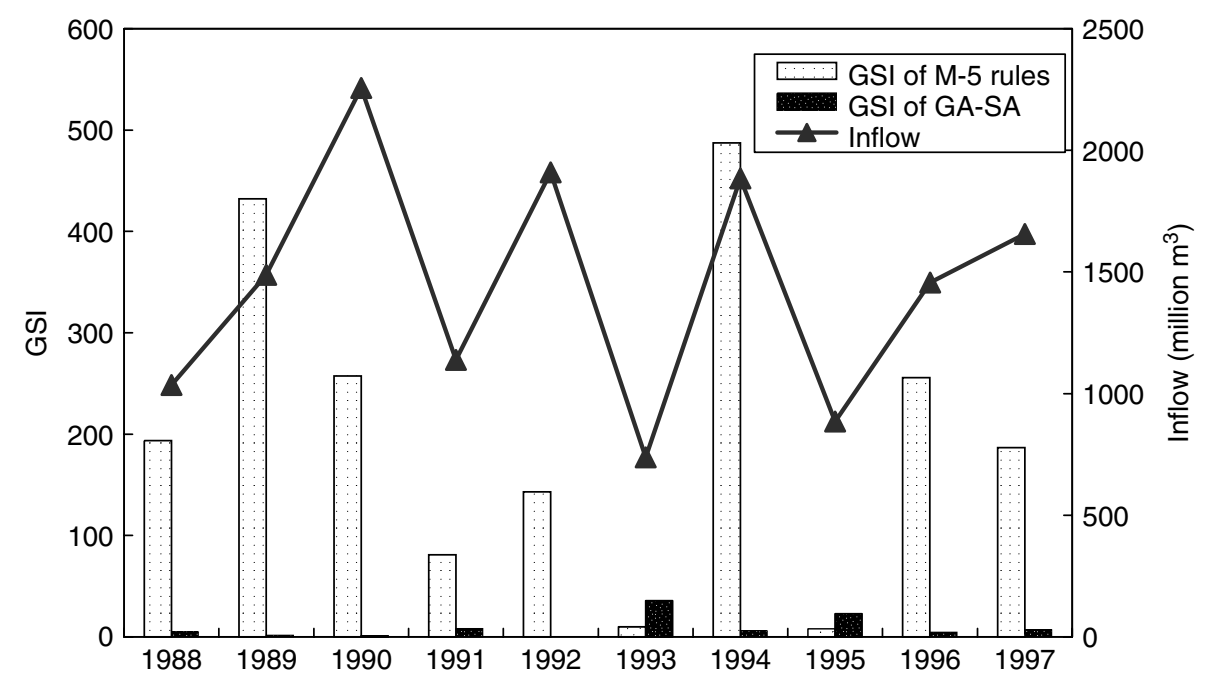

Figure 7. The inflow series and GSI values by the M-5 rules and the GA-SA method in 10 years

degree of satisfaction among the objectives and constraints. Three natural-based evolutional search methods (GA, SA and a hybrid GA-SA) were used to search this complex and non-linear system to determine the optimal schedule of water release and storage for three years with different hydrological conditions. Based on our computation results, the following conclusions can be drawn.

1. The degree of satisfaction proposed in fuzzy programming is a suitable criterion to evaluate the reservoir operation with regard to water deficit and dam safety.

2. All three natural-based search methods have a superior performance, with regard to the high degree of satisfaction and lower GSI index, than the current M-5 rule curves approach in all cases.

3. The GA is more efficient than SA, whereas SA might have a better performance than the GA after a long search iteration. The proposed hybrid GA-SA method achieves the highest satisfaction and lowest GSI values in all cases. The hybrid GA-SA can avoid a long and ineffective searching process in SA at the beginning stages and improve the solution quality of the GA.

Further evaluation of the hybrid GA-SA method was undertaken by searching the long-term reservoir operation performance; we implemented the method using a consecutive 10-year period and compared the performance with the M-5 rule curves simulation. The results again demonstrate that the hybrid GA-SA method indeed outperforms the M-5 operating rule curves in terms of much higher degree of satisfaction and lower GSI values. These results provide further evidence to support that the hybrid GA-SA method is a robust and efficient way for optimizing variables in a highly non-linear problem, such as water resources management.

\section{ACKNOWLEDGEMENTS}

This paper is based on partial work supported by National Science Council, ROC (grant no. NSC91-2313-B-002$315)$.

\section{REFERENCES}

Archibald TW, McKinnon KIM, Thomas LC. 1996. An aggregate stochastic dynamic programming model of multireservoir systems. Water Resources Research 33: 333-340.

Bellman R. 1957. Dynamic Programming. Princeton University Press: Princeton, NJ.

Bellman RE, Zadeh LA. 1970. Decision making in a fuzzy environmental. Management Science B 17: 141-164.

Chang FJ, Chen L. 1991. Investigation of the objection functions for realtime reservoir operation. Taiwan Water Conservancy Quarterly 39(2): 50-58.

Chang FJ, Chen L. 1998. Real-coded genetic algorithm for rule-based flood control reservoir management. Water Resources Management 12: $185-198$.

Chang FJ, Hui SC, Chen YC. 2002. Reservoir operation using grey fuzzy stochastic dynamic programming. Hydrological Processes 16: 2395-2408.

Chang FJ, Chen L, Chang LC. 2005. Optimizing the reservoir operation rule curves by genetic algorithms. Hydrological Processes 19: 2277-2289.

Chang LC, Chang FJ. 2001. Intelligent control for modelling of real-time reservoir operation. Hydrological Processes 15: 1621-1634. 
Chaves P, Kojiri T, Yamashiki Y. 2003. Optimization of storage reservoir considering water quantity and quality. Hydrological Processes 17: 2769-2793.

Datta B, Houck MH. 1984. A stochastic optimization model for real-time operation of reservoirs using uncertain forecasts. Water Research 20: 1039-1064.

Dougherty DE, Marryott RA. 1991. Optimal groundwater management -1. Simulated annealing. Water Resources Research 27: 2493-2508.

Dubrovin T, Jolma A, Turunen E. 2002. Fuzzy model for real-time reservoir operation. Journal of Water Resources Planning and Management 128: 66-73.

Eshelman LJ, Schaffer JD. 1993. Real-coded genetic algorithms and interval-schemata. In Foundations of Genetic Algorithms 2, Whitley LD (ed.). Morgan Kaufmann: San Mateo, CA; 187-202.

Goldberg DE. 1989. Genetic Algorithms in Search, Optimization, and Machine Learning, 2nd edn. Addison-Wesley: Reading, MA.

Hall WA, Buras N. 1961. The dynamic programming approach to water resources development. Journal of Geophysical Research 66: 517-520.

Holland JH. 1992. Adaptation in Natural and Artificial Systems. MIT Press: Cambridge, MA

Hsu SK. 1995. Shortage indices for water-resources planning in Taiwan. Journal of Water Resources Planning and Management 121: 119-131.

Kirpatrick S, Gelatt CD, Vecchi MP. 1983. Optimization by simulated annealing. Science 220(4598): 671-680.

Lin FT, Kao CY, Hsu CC. 1993. Applying the Genetic approach to simulated annealing in solving some NP-hard problems. IEEE Transactions on Systems, Man, and Cybernetics 23: 1752-1767.
Maskey S, Guinot V, Price RK. 2004. Treatment of precipitation uncertainty in rainfall-runoff modelling: a fuzzy set approach. Advances in Water Resources 27: 889-898.

Mousavi SJ, Mahdizadeh K, Afshar A. 2004. A stochastic dynamic programming model with fuzzy storage states for reservoir operations. Advances in Water Resources 27: 1105-1110.

Oliveira R, Loucks DP. 1997. Operating rules for multireservoir systems. Water Resources Research 33: 839-852.

Pardo-Igúzquiza E. 1998. Optimal selection of number and location of rainfall gauges for areal rainfall estimation using geostatistics and simulated annealing. Journal of Hydrology 210: 206-220.

Trezos T, Yeh WW-G. 1987. Use of stochastic dynamic programming for reservoir management. Water Resources Research 23: 983-996.

Wang DW. 1997. An inexact approach for linear programming problems with fuzzy objective and resources. Fuzzy Sets and Systems 89: 61-68.

Wang PP, Zheng C. 1998. An efficient approach for successively perturbed groundwater models. Advances in Water Resources 21: 499-508.

Wardlaw R, Sharif M. 1999. Evaluation of genetic algorithms for optimal reservoir system operation. Journal of Water Resources Planning and Management 125: 25-33.

Yu PS, Chen CJ, Chen SJ. 2000. Application of gray and fuzzy methods for rainfall forecasting. Journal of Hydrologic Engineering 5: 339-345.

Zadeh LA. 1965. Fuzzy sets. Information and Control 8: 338-353.

Zimmermann HJ. 1976. Description and optimization of fuzzy systems. International Journal of General Systems 2: 209-215. 